\title{
Surface characterization of dialyzer polymer membranes by imaging ToF-SIMS and quantitative XPS line scans
}

Markus Holzweber, Andreas Lippitz, Katharina Krueger, Joachim Jankowski, and Wolfgang E. S. Unger

Citation: Biointerphases 10, 019011 (2015); doi: 10.1116/1.4907937

View online: https://doi.org/10.1116/1.4907937

View Table of Contents: http://avs.scitation.org/toc/bip/10/1

Published by the American Vacuum Society

\section{Articles you may be interested in}

Latest applications of 3D ToF-SIMS bio-imaging

Biointerphases 10, 018902 (2015); 10.1116/1.4907727

Multivariate ToF-SIMS image analysis of polymer microarrays and protein adsorption

Biointerphases 10, 019005 (2015); 10.1116/1.4906484

Assessment of different sample preparation routes for mass spectrometric monitoring and imaging of lipids in bone cells via ToF-SIMS

Biointerphases 10, 019016 (2015); 10.1116/1.4915263

3D chemical characterization of frozen hydrated hydrogels using ToF-SIMS with argon cluster sputter depth profiling

Biointerphases 11, 02 A301 (2016); 10.1116/1.4928209

Imaging XPS and photoemission electron microscopy; surface chemical mapping and blood cell visualization

Biointerphases 12, 02 C408 (2017); 10.1116/1.4982644

Full membrane spanning self-assembled monolayers as model systems for UHV-based studies of cellpenetrating peptides

Biointerphases 10, 019009 (2015); 10.1116/1.4908164

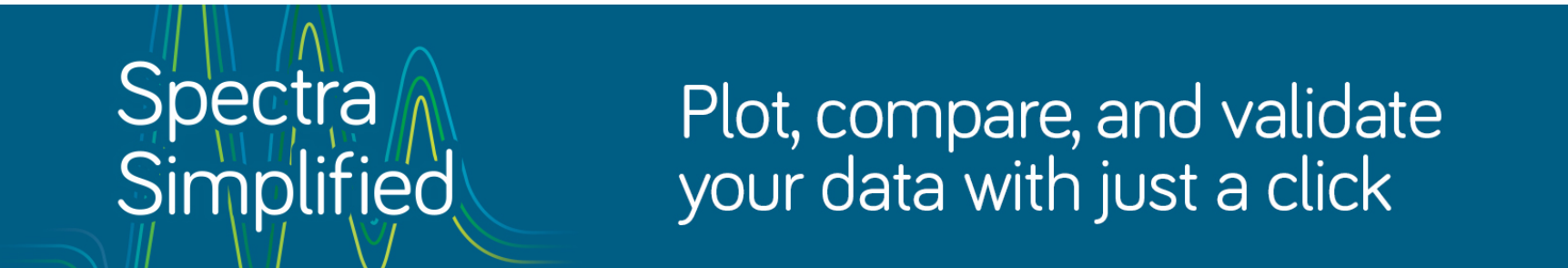

\section{eSpectra:}




\title{
Surface characterization of dialyzer polymer membranes by imaging ToF-SIMS and quantitative XPS line scans
}

\author{
Markus Holzweber ${ }^{a)}$ and Andreas Lippitz \\ Division 6.8-Surface Analysis and Interfacial Chemistry, BAM-Federal Institute for Materials Research \\ and Testing, Unter den Eichen 44-46, 12203 Berlin, Germany \\ Katharina Krueger and Joachim Jankowski \\ Institute for Molecular Cardiovascular Research, University Hospital RWTH Aachen, Pauwelsstrasse 30, \\ 52074 Aachen, Germany \\ Wolfgang E. S. Unger \\ Division 6.8 - Surface Analysis and Interfacial Chemistry, BAM—Federal Institute for Materials Research \\ and Testing, Unter den Eichen 44-46, 12203 Berlin, Germany
}

(Received 15 December 2014; accepted 29 January 2015; published 24 February 2015)

The surfaces of polymeric dialyzer membranes consisting of polysulfone and polyvinylpyrrolidone were investigated regarding the lateral distribution and quantitative surface composition using time-of-flight secondary-ion-mass-spectrometry and x-ray photoelectron spectroscopy. Knowledge of the distribution and composition on the outer surface region is of utmost importance for understanding the biocompatibility of such dialyzer membranes. Both flat membranes and hollow fiber membranes were studied. (C) 2015 Author(s). All article content, except where otherwise noted, is licensed under a Creative Commons Attribution 3.0 Unported License. [http://dx.doi.org/10.1116/1.4907937]

\section{INTRODUCTION}

A very common synthetic polymer used for the production of dialyzer membranes for medical applications is polysulfone (PS). ${ }^{1,2}$ Usually, a hydrophilic modifier, e.g., polyvinylpyrrolidone (PVP), has to be added to PS. The use of PS and PVP results in polymer membranes with excellent biocompatibility and well-defined pore-structure with a fine pored luminal and a wide pored abluminal surface. The luminal surface (blood facing side) corresponds to the inner surface of the dialysis hollow fiber membrane; the outer surface of a dialysis hollow fiber is its abluminal (side facing away from the blood) surface.

For characterization of polymers, a wide spectrum of analytical techniques is available. In general, several techniques have to be combined to provide a detailed polymer characterization. ${ }^{3}$ Although the polymer distribution is essential for both biocompatibility and efficiency, the distribution of the membrane compounds has not been well characterized until now. In recent years, mass spectrometry (MS), in particular, matrix-assisted laser desorption/ionization MS (MALDI) and secondary ion mass spectrometry (SIMS) techniques, has been established as powerful tool for characterization of polymers and other organic materials and coatings. In the imaging mode, these MS techniques enable both identification of compounds as well as their localization and distribution.

The spatial distribution of polymers in biomaterials is essential for biocompatibility of these materials. For example, the distribution of PVP on PS is crucial for the

\footnotetext{
${ }^{a}$ Author to whom correspondence should be addressed; electronic mail: markus.holzweber@bam.de
}

biocompatibility of dialyzer membranes. The lateral and indepth detection of the polymers is essential for optimizing the biocompatibility of medical products used for dialysis. Exposure of blood to hemodialysis membranes results in numerous interactions between blood cellular components and membrane surface having the potential to induce an inflammatory response and to lead to numerous long-term clinical sequel that are in part determined by the degree of membrane biocompatibility. A biocompatible membrane has traditionally been defined as "one that elicits the least amount of inflammatory response in patients exposed to $i t . "{ }^{\prime, 5}$ The Consensus Conference on Biocompatibility recommended the following criteria to assess material biocompatibility:

(1) evaluation of thrombosis formation upon contact of blood with the artificial material

(2) signs of coagulation system activation

(3) signs of thrombocyte activation.

Polysulfone-based membranes are widely used for production of conventional hemodialysis membranes since these membranes fulfill most of these criteria. ${ }^{5}$

Polysulfone-based biomaterials are presently the gold standard in the production of biocompatible hemodialyzers. As polysulfone-based biomaterials are hydrophobic, these membranes have to be blended with hydrophilic polymers, such as polyvinylpyrrolidone ${ }^{6}$ for increasing biocompatibility and wettability. The resulting membranes have to be characterized by physical and chemical techniques regarding membrane structure, defined in terms of size, form, and distribution of the pores at the luminal separating layer. ${ }^{6}$ Second, the physical and chemical properties of the blood contacting surface should minimize the interaction of blood 
and biomaterial neither affecting the membrane function nor causing adverse reactions for the patient. However, up to now, there has been no standard technique so far for the measurement of compounds like polyvinylpyrrolidone on the luminal and abluminal membrane sides available. As a result, the biocompatibility is solely determined by physiologic assays without the possibility to analyze and optimize the distribution of these compounds by an analytic method before using the membranes in physiologic assays. Time-offlight (ToF) SIMS and MALDI MS imaging are useful and complementary tools for molecular characterization of polymer membrane surfaces. After the investigation of the measurement capability of imaging MALDI-MS (Ref. 4) we here report a parallel study where imaging ToF-SIMS and quantitative $\mathrm{x}$-ray photoelectron spectroscopy (XPS) have been used to characterize the very surface of PS/PVP dialyzer membrane samples.

\section{EXPERIMENT}

\section{A. Preparation of the membranes}

The preparation of the flat membrane is described elsewhere. ${ }^{4}$ Briefly, a solution of $15 \%$ polysulfone and $3.75 \%$ polyvinylpyrrolidone in dimethylacetamide (DMAc) was spread out by a scalpel and precipitated by a mixture of $70 \%$ DMAc and 30\% water (structures of PS and PVP are given in Fig. 1). The freshly formed membrane was incubated in distilled water overnight and dried subsequently.

The hollow fiber membrane originates from a commercially available hemodiafilter (Fresenius Helixone ${ }^{\circledR}$ FX 800 HDF). The hollow fiber was opened using a sterile scalpel and was prepared as a flat specimen on a double sided adhesive tape for subsequent analysis.

\section{B. Time-of-flight secondary-ion-mass-spectrometry}

All sample measurements were performed on a ToF.SIMS IV instrument (ION-TOF GmbH, Münster, Germany) of the reflectron-type, equipped with a $25 \mathrm{keV}$ bismuth liquid metal ion gun (LMIG) as primary ion source mounted at $45^{\circ}$ with respect to the sample surface. The

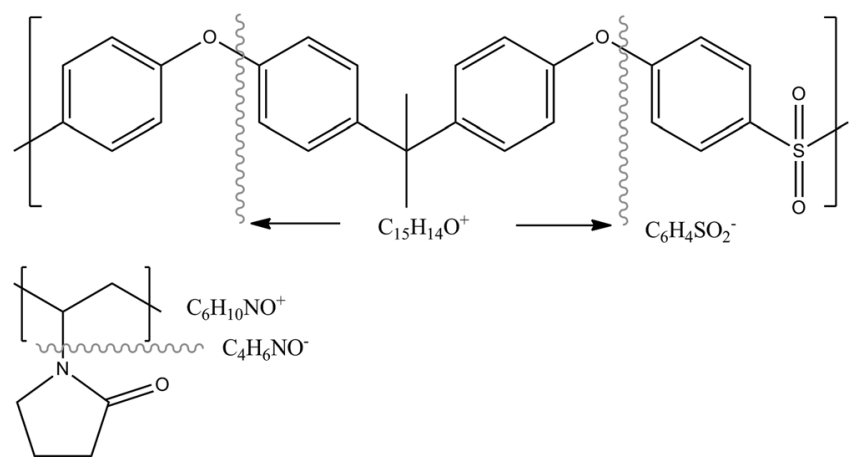

FIG. 1. Structures of polymers PS (top) and PVP polymers (bottom) with characteristic secondary ion fragments in the positive and negative ion detection mode used for imaging.
LMIG was operated at $0.5 \mu \mathrm{A}$ emission current in the socalled "high current bunched" mode (high mass resolution, low lateral resolution). $\mathrm{Bi}_{3}{ }^{+}$was selected as primary ion by appropriate mass filter settings. To improve the focus of the primary ion beam (and hence the lateral resolution) the pulse width of the $\mathrm{Bi}_{3}{ }^{+}(25 \mathrm{keV})$ ion pulse was reduced to $11 \mathrm{~ns}$ and the lens target was adjusted to obtain a sharp image on a structured sample (e.g., silver cross) in the secondary electron mode. The primary ion current was directly determined at $100 \mu$ s cycle time (i.e., a repetition rate of $10 \mathrm{kHz}$ ) using a Faraday cup located on a grounded sample holder. Operation conditions with these settings comprised a target current of $0.19 \mathrm{pA}$ for the selected primary ion. The large area scans (3 $\times 3 \mathrm{~mm}$ ) were performed in the so-called stitching mode where the total analysis area is divided into several small analysis areas, which are stitched together by a routine implemented in the SurfaceLab 6.4 (ION-TOF GmbH, Münster, Germany) software. The total primary ion dose density was $5 \times 10^{10}$ ions $/ \mathrm{cm}^{2}$ for large area scans and 5.1 $\times 10^{11}$ ions $/ \mathrm{cm}^{2}$ for a standard measurement area $(500 \times 500$ $\mu \mathrm{m}$ ) ensuring static conditions. ${ }^{7}$ The vacuum in the analysis chamber was in the range of $10^{-9}$ mbar during all measurements.

ToF-SIMS spectra were acquired both in positive and negative ion detection mode from identical analysis areas. The mass scale was internally calibrated using a number of well defined and easily assignable secondary ions $\left(\mathrm{C}_{4} \mathrm{H}_{2}{ }^{+}, \mathrm{C}_{5} \mathrm{H}_{5}{ }^{+}, \mathrm{C}_{6} \mathrm{H}_{5}{ }^{+}, \mathrm{C}_{7} \mathrm{H}_{7}{ }^{+}, \mathrm{C}_{9} \mathrm{H}_{11} \mathrm{O}^{+}, \mathrm{C}_{15} \mathrm{H}_{14} \mathrm{O}^{+}\right.$, and $\mathrm{C}_{27} \mathrm{H}_{23} \mathrm{SO}_{4}^{+}$for positive ion mode and $\mathrm{HS}^{-}, \mathrm{SO}^{-}$, $\mathrm{C}_{4} \mathrm{H}_{6} \mathrm{NO}^{-}, \mathrm{C}_{6} \mathrm{H}_{3} \mathrm{SO}_{2}{ }^{-}, \mathrm{C}_{14} \mathrm{H}_{11} \mathrm{O}_{2}{ }^{-}$, and $\mathrm{C}_{21} \mathrm{H}_{17} \mathrm{SO}_{4}{ }^{-}$for negative ion mode) keeping the error in calibration for the selected secondary ions below $10 \mathrm{ppm}$.

\section{X-ray photoelectron spectroscopy}

XPS measurements for the flat membrane were performed on an ESCALab 200X photoelectron spectrometer (VG Scientific, UK). XPS survey spectra were recorded at an angle of emission of $0^{\circ}$ to the surface normal using nonmonochromatized $\mathrm{Al} \mathrm{K} \alpha$ excitation at the CRR 10 mode. Quantification was carried out using a routine implemented in the Avantage 4.43 software. The routine uses Scofield's photoionization cross sections for determination of relative sensitivity factors. This quantification routine uses the model of homogeneous distribution of all constituents in the analyzed volume, which is limited by an information depth of around $10 \mathrm{~nm}$.

XPS measurements for the hollow fiber membrane were carried out with an AXIS Ultra DLD electron spectrometer (Kratos Analytical, UK). XPS spectra were recorded using monochromated $\mathrm{Al} \mathrm{K} \alpha$ excitation at pass energy of $80 \mathrm{eV}$ for survey spectra. The electron emission angle was $0^{\circ}$ to the surface normal and the source-to-analyzer angle was $60^{\circ}$. Spectra were taken by setting the instrument to the hybrid lens mode and the slot mode providing approximately an analysis area of $300 \times 700 \mu \mathrm{m}^{2}$. Quantification was carried out using a routine implemented in the CASA 
positive ion mode

b)
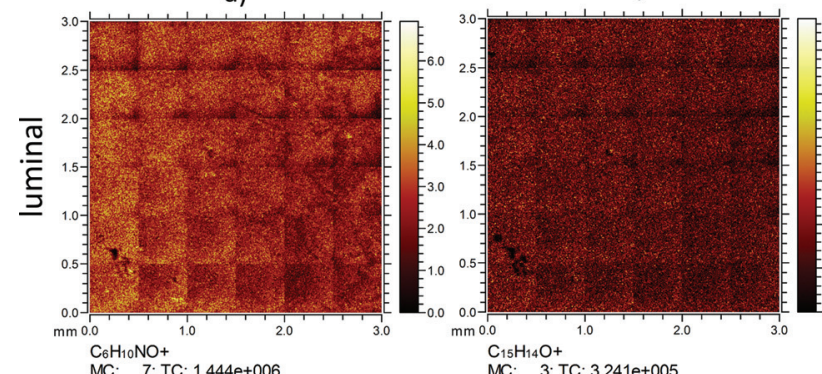

MC: $\quad 3 ;$ TC: $3.241 \mathrm{e}+005$

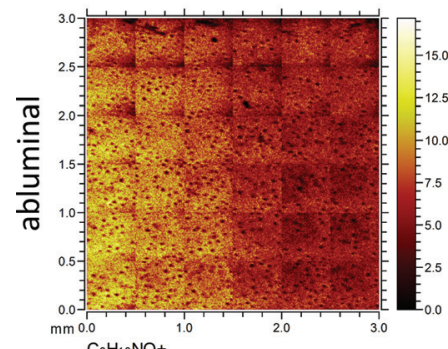

MC: $17 ;$ TC: 4.011 e+006

d)
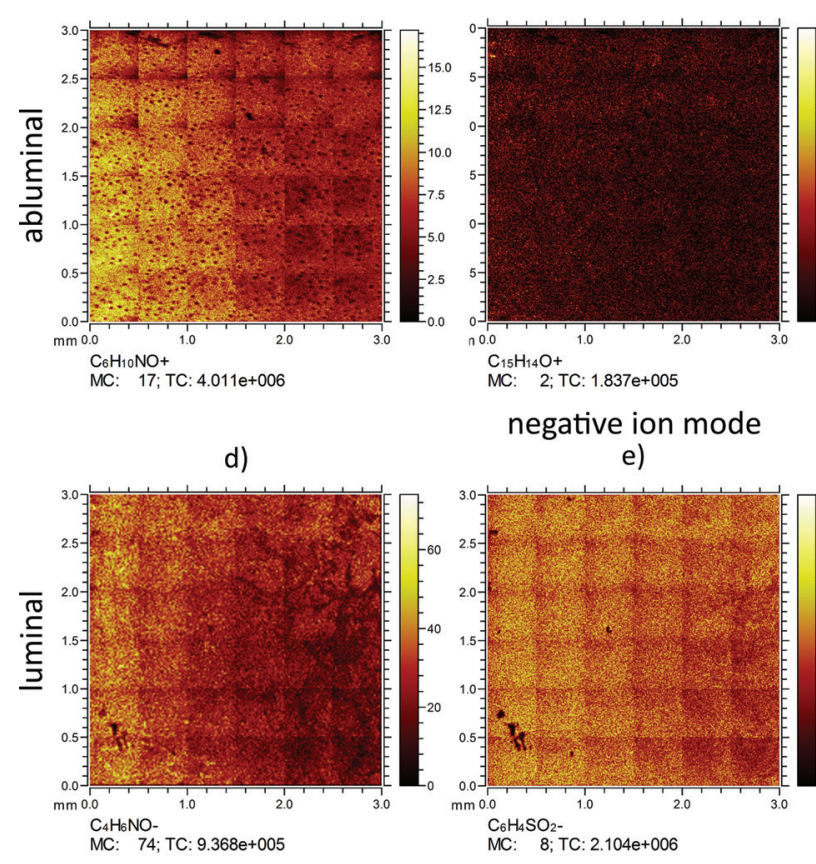

negative ion mode

e)
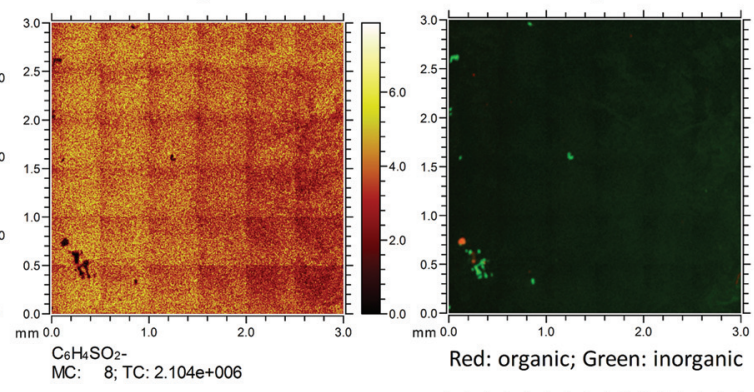

Red: organic; Green: inorganic

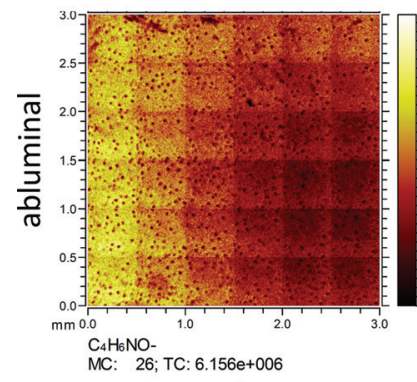

g)

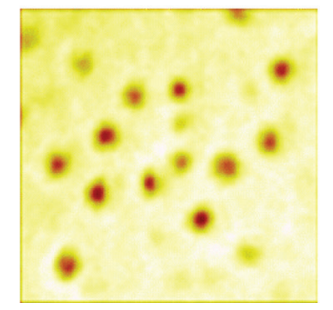

c)

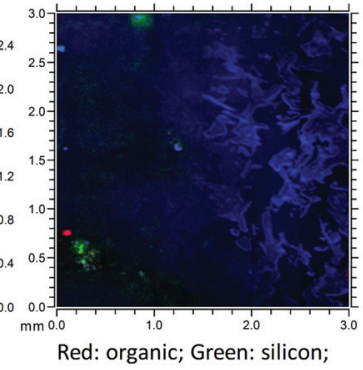

Blue: inorganic

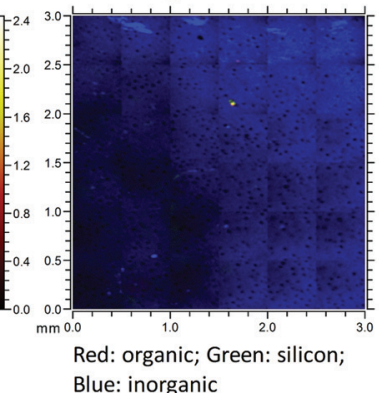

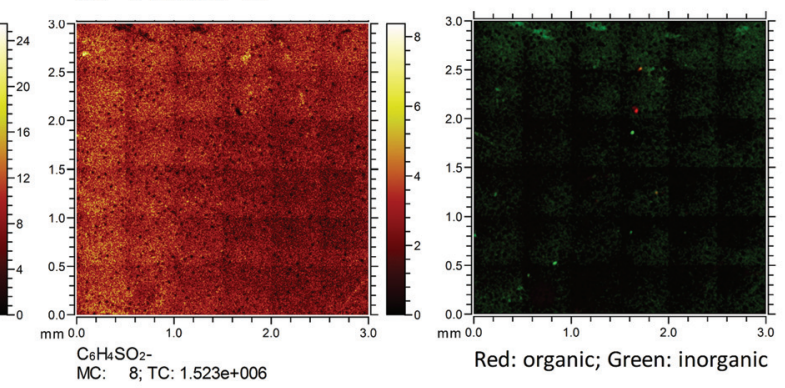

FIG. 2. Secondary ion macroimages $(3 \times 3 \mathrm{~mm})$ showing the lateral distribution of characteristic fragment secondary ions for $\mathrm{PVP}$ and $\mathrm{PS}$ (a) $\mathrm{C}_{6} \mathrm{H}_{10} \mathrm{NO}^{+}$, (b) $\mathrm{C}_{15} \mathrm{H}_{14} \mathrm{O}^{+}$, and (c) preparation residues (RGB overlay) in positive ion mode and (d) $\mathrm{C}_{4} \mathrm{H}_{6} \mathrm{NO}^{-}$, (e) $\mathrm{C}_{6} \mathrm{H}_{4} \mathrm{SO}_{2}^{-}$, (f) preparation residues (RGB overlay) in negative ion mode for the luminal and the abluminal side of the flat membrane and (g) a $400 \times 400 \mu \mathrm{m}$ square detail from the total ion image of the abluminal side showing the pores as dark spots. 
positive ion mode

a)
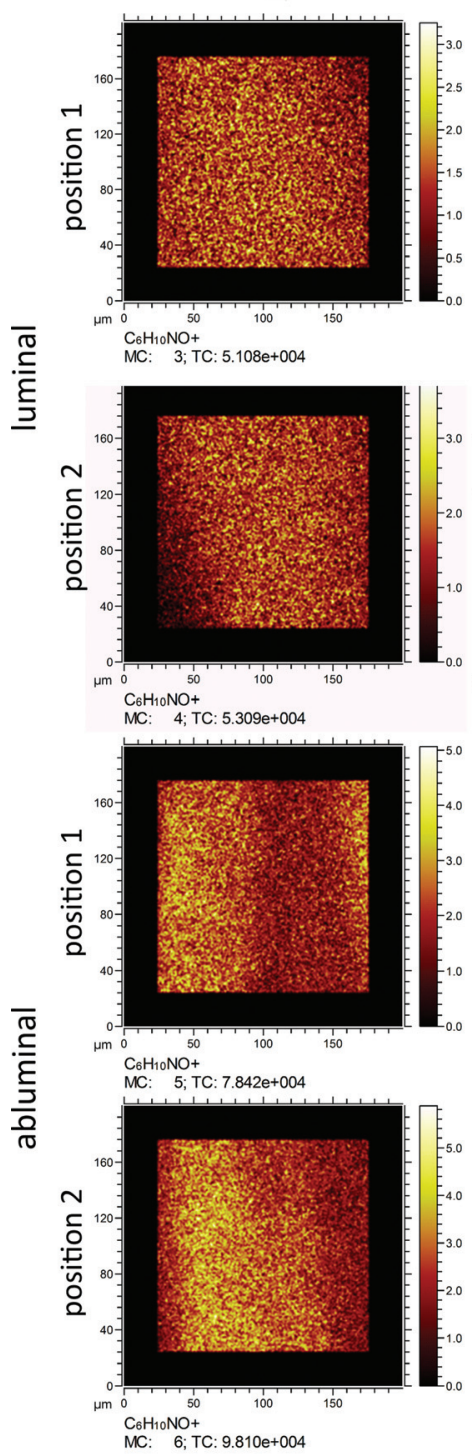

b)

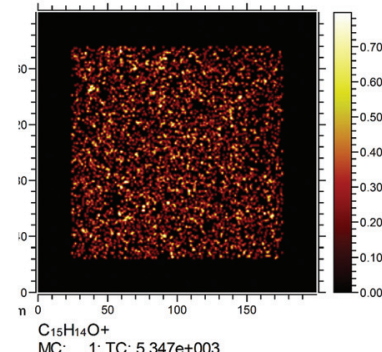

MC. 1 TC. 5347 e+003
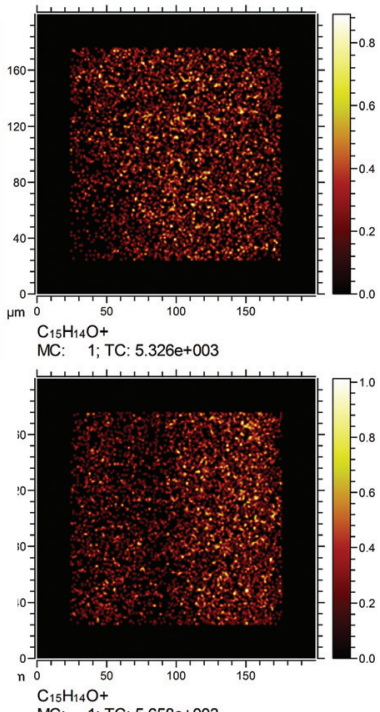

MC: $1 ;$ TC: $5.658 \mathrm{e}+003$

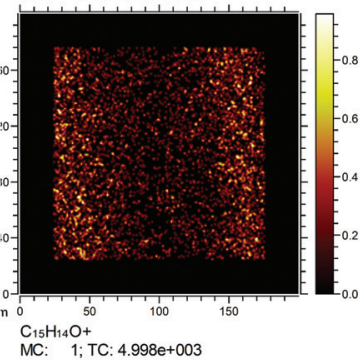

negative ion mode

c)
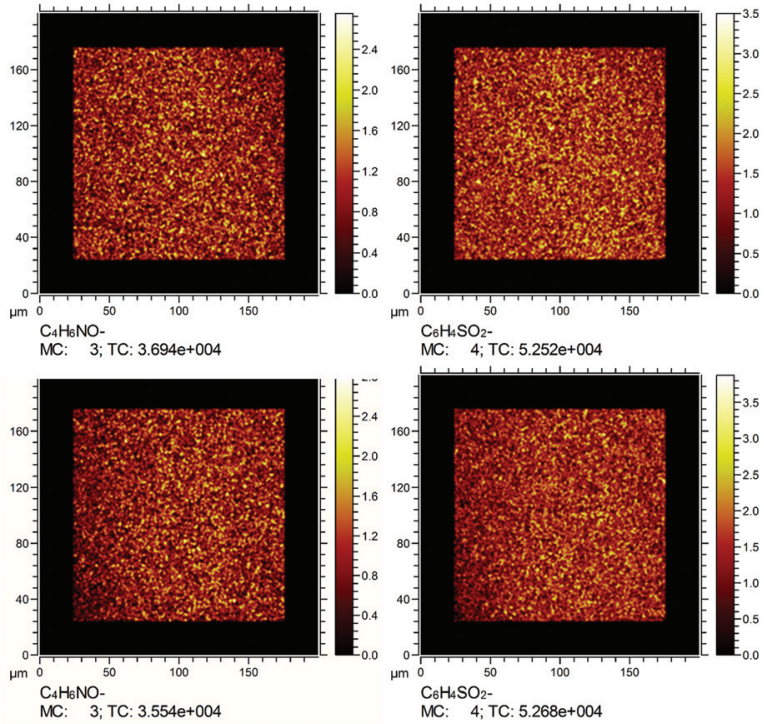

$\mathrm{C}_{4} \mathrm{H}_{6} \mathrm{NO}-$
$\mathrm{MC}: \quad 3 ; \mathrm{TC}: 3.554 \mathrm{e}+004$
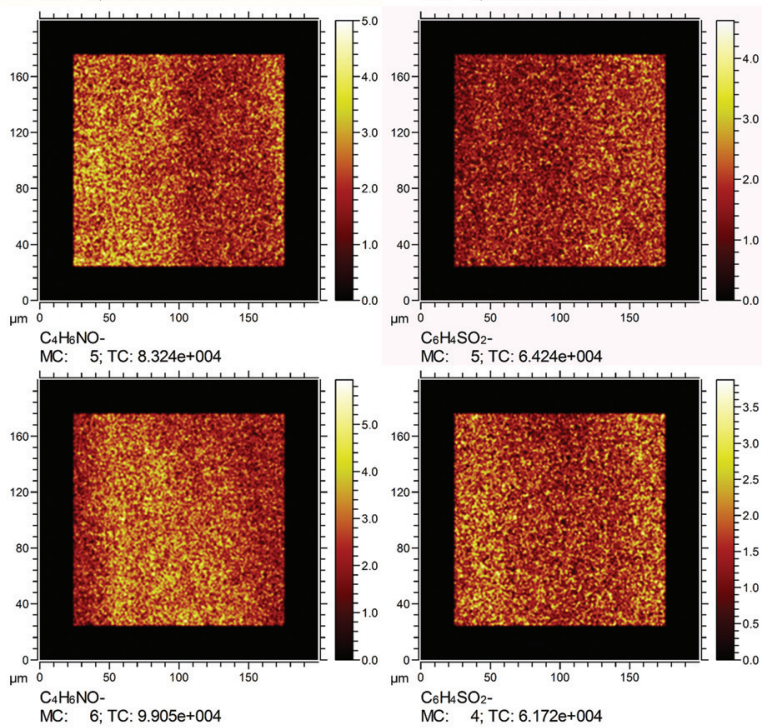

d)

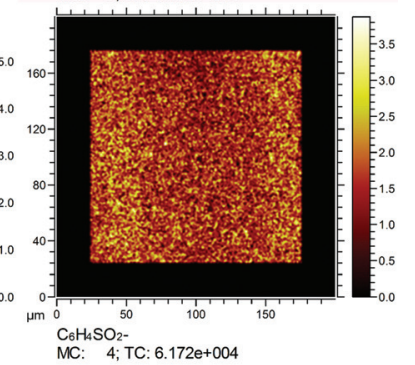

FIg. 3. Secondary ion images showing the lateral distribution of characteristic fragment ions for PVP and PS for two distinct measuring positions. (a) $\mathrm{C}_{4} \mathrm{H}_{6} \mathrm{NO}^{+}$, (b) $\mathrm{C}_{15} \mathrm{H}_{14} \mathrm{O}^{+}$in positive ion mode and (c) $\mathrm{C}_{4} \mathrm{H}_{6} \mathrm{NO}^{-}$, (d) $\mathrm{C}_{6} \mathrm{H}_{4} \mathrm{SO}_{2}^{-}$in negative ion mode for the luminal (top) and the abluminal (bottom) side of the hollow fiber membrane.

XPS software. This routine uses sensitivity factors from the Kratos library.

The binding energy (BE) scale was calibrated in each case using the aliphatic hydrocarbon $\mathrm{C} 1 \mathrm{~s}$ component at $\mathrm{BE}$ $=285.0 \mathrm{eV}$.

\section{RESULTS}

\section{A. Lateral distribution of the polymer constituents on the investigated surfaces as revealed by imaging ToF-SIMS}

In a previous study, specimens of the same batch were analyzed by imaging MALDI to investigate the chemical structure and the distribution of the membrane compounds. ${ }^{4}$ In that paper, the essential parameters laser intensity and raster step size were systematically varied in MALDI-imaging analysis in order to measure distributions of the polymeric components on both sides at optimized settings of the MALDI experiment (grid distances for laser spot sample preparation and laser intensity). For the abluminal side of the flat membrane MALDI reveals a homogenous distribution of PS but PVP condensed in patches. At the luminal side PVP was more homogenously distributed than PS. In the case of the hollow fiber membrane PS is more present at the abluminal side and more homogenously distributed. The luminal side showed PVP to be more enriched and more evenly distributed. In both cases, a different polymeric composition on the luminal and abluminal sides has been concluded. It was attractive to use much more surface sensitive methods as XPS and ToF-SIMS (information depths $\sim 10$ and $\sim 3 \mathrm{~nm}$, 
position 1
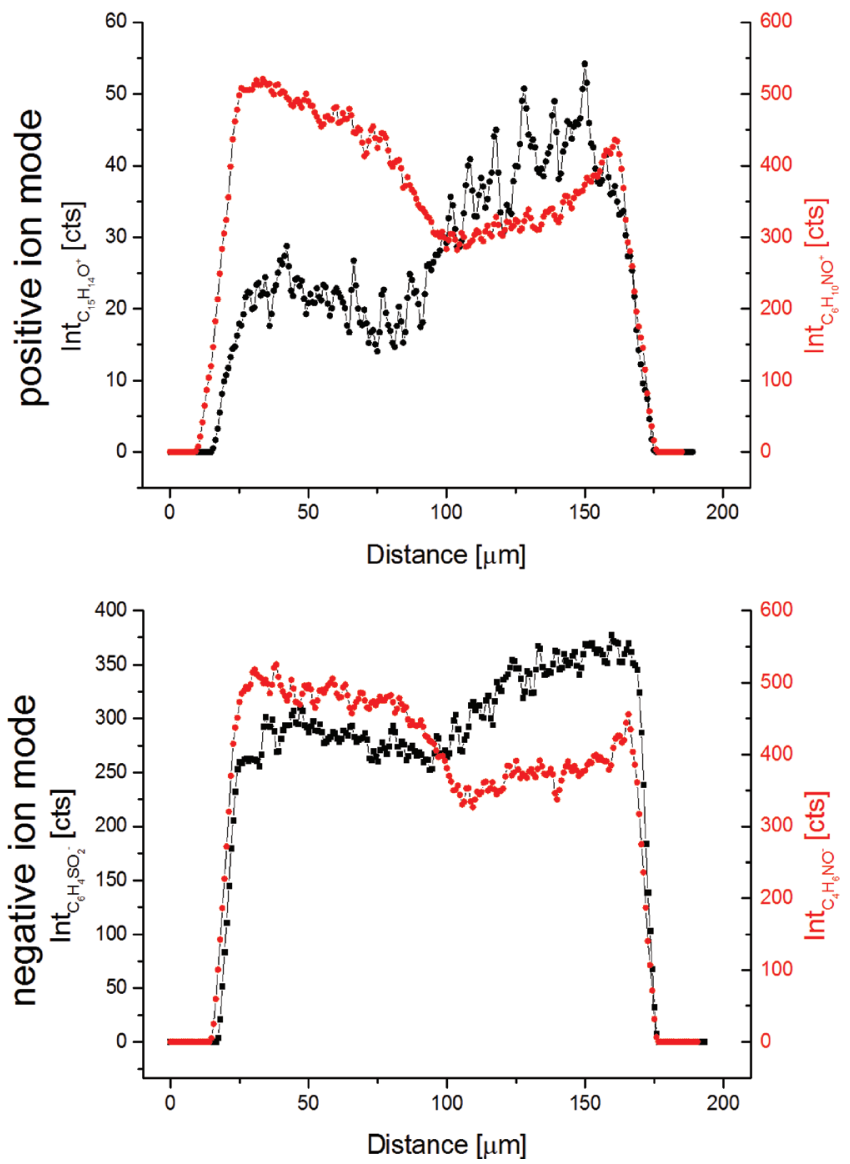

position 2
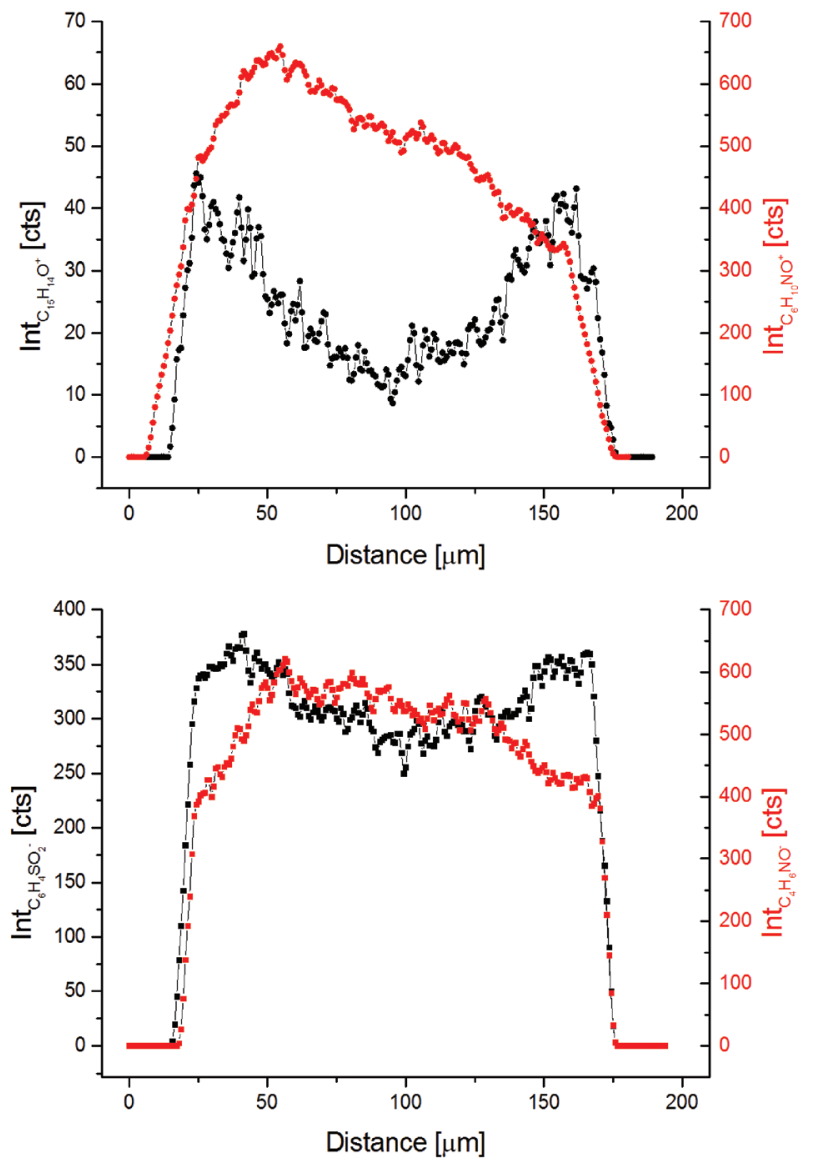

FIG. 4. Area scans from the abluminal side of the hollow fiber showing the intensity distribution of the characteristic polymer signals in positive and negative ion detection mode for both measuring positions.

respectively) to analyze the luminal and abluminal surfaces of both samples. These results are highly relevant for the interaction of blood cells with the membrane material.

In our SIMS study, surface sensitive information on composition and distribution of the polymers is revealed. Figure 2 shows images of the distribution of characteristic fragment ions for the polymers PVP and PS for the flat membrane from identical scanning areas in positive and negative ion mode. As characteristic secondary fragment ions for PVP the pyrrilidon side group, $\mathrm{C}_{6} \mathrm{H}_{10} \mathrm{NO}^{+}$, and $\mathrm{C}_{4} \mathrm{H}_{6} \mathrm{NO}^{-}$, at the polymer chain were chosen in positive and negative ion detection mode, respectively. As characteristic fragment secondary ions for the PS repetition unit $\mathrm{C}_{15} \mathrm{H}_{14} \mathrm{O}^{+}$(originating from the bisphenol-A moiety of PS) and $\mathrm{C}_{6} \mathrm{H}_{4} \mathrm{SO}_{2}{ }^{-}$were selected (for structures see Fig. 1). Relying on these fragment ions, it can clearly be stated that the PVP and PS are both homogeneously distributed on both the luminal and abluminal surface of the flat membrane.

Other features in the SIMS images have to be addressed, too. The randomly distributed "dots" in the image of the abluminal side of the flat membrane correspond to its pores [see Fig. 2(g)] in the size range of up to $50 \mu \mathrm{m}$ as revealed by SEM [cf. SEM images in Fig. 2(a) left in Ref. 4] causing a shadowing effect for the primary ions impinging at an angle of $45^{\circ}$. Pores on the luminal side are in the sub-micrometer range $(20-60 \mathrm{~nm})$ (cf. SEM images in Fig. 2(a) right in Ref. 4], which is well below the ToFSIMS lateral resolution in the used measuring mode $(1-3 \mu \mathrm{m}$ lateral resolution ${ }^{8}$ ). The dark areas in the secondary ion images in Fig. 2 are related to preparation residues $(\mathrm{Na}, \mathrm{K}, \mathrm{Ca}$, polydimethylsiloxane, and some unclear organic species presumably some remains from the used solvents) on top of the flat membrane surface, which are highlighted by the RGB overlays in Fig. 2. Also, some minor charging effects can be seen on the patch borders of the stage scan which have a negligible influence on the data interpretation is this case.

The hollow fiber dialyzer membrane was investigated also with respect to lateral distribution of PS and PVP on both surfaces. Figure 3 displays the distribution of the characteristic PVP and PS fragments on the analyzed surface. To reduce topographic effects from the edges of the scanning area a center square of the image is reconstructed from the raw data for subsequent interpretation. This effect can be recognized by a decrease in signal intensity for both characteristic polymer (PVP and PS) fragments [see Fig. 3(a), luminal position 2]. For the luminal side, the secondary ion images reveal a homogeneous lateral distribution of the polymeric constituents at the scale of lateral resolution of SIMS as observed for the flat membrane sample before. The pore size at both sides of the hollow fiber dialyzer membrane is in the low-micrometer to sub-micrometer range [cf. SEM 

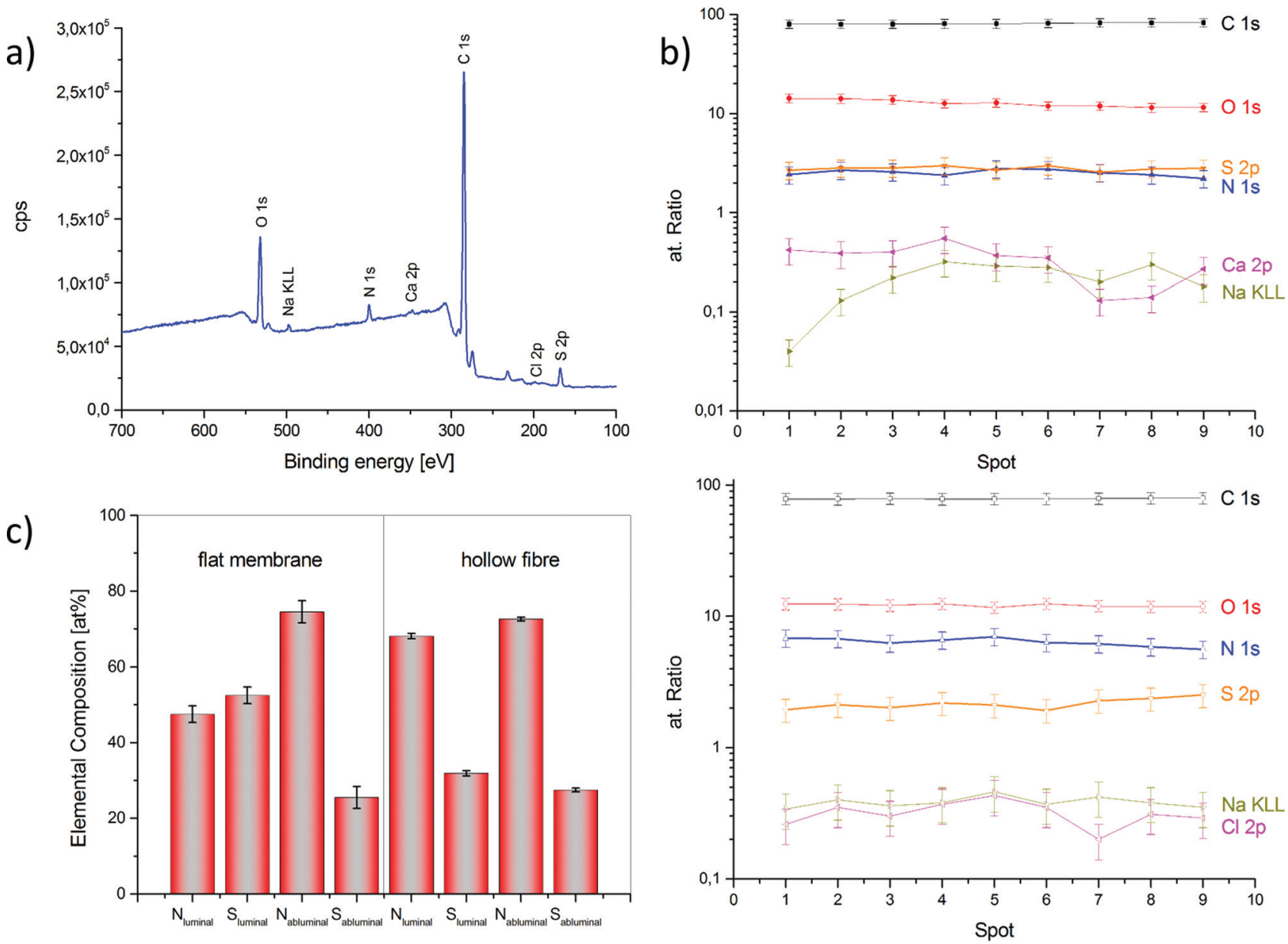

FIG. 5. (a) Typical XPS survey spectrum showing the main constituents $\mathrm{C}, \mathrm{N}, \mathrm{S}$, and $\mathrm{O}$ as well as some minor preparation residues as Na, Ca, and $\mathrm{Cl}$. (b) Line scan over the flat membrane showing the surface concentrations at several measurement spots for the main constituents of the polymers and the preparation residues; top: luminal side, bottom: abluminal side. Error bars are expert based estimations of uncertainty of measurement ( $\pm 10 \%$ for main, $\pm 20 \%$ for minor, and $\pm 30 \%$ for trace components). (c) Comparison of the surface concentrations measured at both sides of the flat membrane and the hollow fiber as well.

images in Fig. 2(b) in Ref. 4], which is again well below the ToF-SIMS lateral resolution.

On the abluminal side of the hollow membrane, a decrease in signal intensity is detected, but the trend is opposing for the characteristic PVP and PS signals. Therefore, this is not a topographic effect but an inconsistency in chemical composition (see Fig. 4) presumably from the hollow fiber production.

\section{B. Surface composition by XPS}

For quantitative analysis, XPS line scans were carried out across both sides of the flat membrane. The measuring spot had a diameter of $5 \mathrm{~mm}$ and nine spots with a step size of $3 \mathrm{~mm}$ were analyzed for both sides. Figure 5(a) shows a typical XPS survey spectrum of the flat dialyzer membrane. The main components $\mathrm{C}, \mathrm{N}, \mathrm{S}$, and $\mathrm{O}$ were found together with minor preparation residues as $\mathrm{Na}(0-0.3$ at. \%) and $\mathrm{Ca}$ $(0.1-0.6$ at. $\%)$ on the luminal side and sodium $(0.3-0.5$ at. $\%)$ and chloride $(0.2-0.4$ at. $\%)$ on the abluminal side. The key elements for PVP and PS are nitrogen and sulfur, respectively. Since there is only one $\mathrm{N}$ or $\mathrm{S}$ per repetition unit of each of the polymer constituents, an intensity ratio of these elements gives directly the polymer composition at the surface. The results of a line scan for both sides of the flat membrane are summarized in Fig. 5(b). The surface concentration of $\mathrm{N}$ and $\mathrm{S}$ is nearly constant (within error bars) on the luminal side and the ratio $\mathrm{I}_{\mathrm{S} / \mathrm{N}}$ is approximately $1: 1$ $\left[\mathrm{S}_{\text {luminal }}(\right.$ at. $\%)=52.5 \pm 2.2 ; \mathrm{N}_{\text {luminal }}($ at. $\left.\%)=47.5 \pm 2.2\right]$. In contrast, on the abluminal side, the content of $\mathrm{N}$ and $\mathrm{S}$ is significantly different and a ratio $\mathrm{I}_{\mathrm{S} / \mathrm{N}}$ of about $1: 3\left[\mathrm{~S}_{\text {abluminal }}\right.$ (at. $\%)=25.5 \pm 2.9 ; \quad \mathrm{N}_{\text {abluminal }} \quad($ at. $\left.\%)=74.5 \pm 2.9\right] \quad$ is found. The polymeric composition of the hollow fiber, however, is nearly identical on both sides with a ratio $\mathrm{I}_{\mathrm{S} / \mathrm{N}}$ of approx. $1: 3 \quad\left[\mathrm{~S}_{\text {luminal }} \quad(\right.$ at. $\%)=31.9 \pm 0.7 ; \mathrm{N}_{\text {luminal }}$ (at. $\%)=68.1 \pm 0.7 ; S_{\text {abluminal }}($ at. $\%)=27.4 \pm 0.5 ; \mathrm{N}_{\text {abluminal }}$ $($ at. $\%)=72.6 \pm 0.5]$. The comparison of the flat membrane and the hollow fiber is summarized in Fig. 5(c).

\section{DISCUSSION}

Upon comparing the results of the previous MALDI study ${ }^{4}$ with the current findings of the ToF-SIMS and XPS study, we see substantial differences in lateral distribution as 
well as surface composition. ToF-SIMS shows no structures or condensed clusters of PVP or PS neither on the flat membrane's nor on the hollow fiber's surfaces. Both polymers are rather homogeneously distributed at each investigated surface at the scale of applied lateral resolution of ToFSIMS, which is estimated to be in the range of $1-3 \mu \mathrm{m}$ (Ref. 8) (depending on the used primary ion). The surface composition of the luminal and abluminal sides differs from those reported in the MALDI study. Whereas MALDI results suggest an enrichment of PVP on the luminal side compared to the abluminal one, quantitative XPS in contrast shows a nearly $1: 1$ ratio of PVP to PS on the luminal side and an enrichment of PVP on the abluminal side of the flat membrane. Also, for the hollow fiber, an enrichment of PVP in the luminal membrane surface was found by MALDI, which is not in agreement with the XPS results, which show a rather similar composition of PVP:PS of 3:1 at both sides.

The reason for the different findings of the MALDI and the ToF-SIMS/XPS studies of the polymeric membrane materials is believed to be a consequence of the different information depths of the used analysis techniques. MALDI analysis samples a volume extending roughly $1 \mu \mathrm{m}$ deep for each laser pulse. For the surface analytical methods ToFSIMS and XPS the information depth is only a few nanometers (ToF-SIMS: $\sim 3 \mathrm{~nm}$, XPS: $\sim 9 \mathrm{~nm}$ ) for polymers. Also, the image resolution in ToF-SIMS, estimated to be in the order of $1-3 \mu \mathrm{m}$ is rather different from the MALDI experiments where a laser spot with a diameter of $\sim 100 \mu \mathrm{m}$ is rastered with grid distances of 100 or $50 \mu \mathrm{m}$ (with overlapping adjacent sample positions in the latter case) across the sample's surface.

\section{CONCLUSION}

Previous analysis of bicomponent dialyzer membranes using MALDI-MS imaging has shown differences in polymer distribution and composition at the abluminal and luminal membrane surfaces. In contrast, analysis of similar samples using ToF-SIMS imaging has shown a homogeneous polymer distribution for all investigated surfaces except the abluminal side of the hollow fiber. Further quantification by XPS revealed a different polymeric composition as determined previously by MALDI-MS. We conclude that MALDI and XPS/ToF-SIMS are complementary methods in terms of information depth. MALDI samples the surface and extended subsurface regions up to several micrometers indepth whereas XPS/ToF-SIMS is addressing the very surface, which is most important for biocompatibility. So, the inhomogeneities reported in the MALDI study might be related to subsurface phenomena in the PVP modified PS material. This assumption could be tested with imaging analysis techniques with an information depth in-between ToFSIMS/XPS and MALDI-MS like Raman microprobe and attenuated total reflection infrared spectroscopy with an information depth up to $2 \mu \mathrm{m}$ for both techniques. ToF-SIMS molecular depth profiling using argon gas cluster ion beams and a subsequent $3 \mathrm{D}$ reconstruction could give further insights to the subsurface composition as well as SEM/TEM with staining to show possible interior phase segregation.

\section{ACKNOWLEDGMENTS}

The authors thank Birgit Hagenhoff from Tascon GmbH Münster for the discussion of the data and proof reading of the manuscript. M.H. is grateful for financial support by the Austrian Science Found (FWF) through the ErwinSchrödinger fellowship program (Project No. J 3471-N28). In addition, J.J. got a financial support by BMBF/IGSTC project "NPORE" (Project No. 01DQ13006A) to perform the study.

${ }^{1}$ R. M. Hakim, P. J. Held, D. C. Stannard, R. A. Wolfe, F. K. Port, J. T. Daugirdas, and L. Agodoa, Kidney Int. 50, 566 (1996).

${ }^{2}$ S. Subramanian, R. Venkataraman, and J. A. Kellum, Kidney Int. 62, 1819 (2002).

${ }^{3}$ M. J. Ariza, P. Prádanos, R. Rico, E. Rodríguez-Castellón, and J. Benavente, Surf. Interface Anal. 35, 360 (2003).

${ }^{4}$ K. Krueger, C. Terne, C. Werner, U. Freudenberg, V. Jankowski, W. Zidek, and J. Jankowski, Anal. Chem. 85, 4998 (2013).

${ }^{5}$ M. P. C. Grooteman and M. J. Nubé, Nephrol. Dial. Transplant. 19, 2965 (2004).

${ }^{6}$ S. K. Bowry, E. Gatti, and J. Vienken, Contrib. Nephrol. 173, 110 (2011).

${ }^{7}$ J. C. Vickerman and D. Briggs, TOF-SIMS: Surface Analysis by Mass Spectrometry (SurfaceSpectra, Manchester and IM Publications, Chichester, 2001), p. 789.

${ }^{8}$ M. Senoner and W. E. S. Unger, J. Anal. At. Spectrom. 27, 1050 (2012). 